\title{
Shape Preservation of Tension Controlled Ternary 4-point Non-Stationary Interpolating Subdivision Schemes
}

\author{
Khurram Prevez ${ }^{* 1}$ and Syed Hussain Shah ${ }^{2}$ \\ ${ }^{1}$ Department of Mathematics, Balochistan University of Information Technology, \\ Engineering \& Management Sciences, Quetta-87300, Pakistan. \\ ${ }^{2}$ Department of Economics, Balochistan University of Information Technology, \\ Engineering \& Management Sciences, Quetta-87300, Pakistan.
}

August 10, 2018

\begin{abstract}
The aim of this work is to analyze and investigate the shape preserving properties of ternary 4-point non-stationary interpolating subdivision schemes constructed by Beccari et al. [1] with a tension parameter $\omega_{k+1}$ which can reproducing exponential. Moreover, the conditions on the initial control points are developed that allow user to generate shape preserving limit curves after a finite number of subdivision steps and generalize these results in limiting case. Significance of derived conditions are illustrated through graphs and the whole discussion is followed by examples.
\end{abstract}

2010 AMS Classification: 40A05, 65D17, 52B55

Keywords and phrases: Ternary subdivision schemes, Non-stationary, Tension controlled, shape preservation

\section{Introduction}

Subdivision schemes have become one of the most essential tool for the generation of smooth curve or surfaces from discrete set of data points. The subdivision schemes have been appreciated in many fields such as computer aided geometric design (CAGD), image processing, animation industries and computer graphics etc. The subdivision technique is an elegant method defines a smooth curve out of an initial control polygon or a surface out of an initial control mesh by subdividing them according to some refining rules recursively.

${ }^{*}$ coorresponding author 


\section{Shape preservtion}

The property of shape preservation is of great practical importance in mod geology, airplane and ship hulls designingeling curves \& surfaces tailored to industrial design such as automobile, aircraft design and ship modeling where convexity is imposed technical and physical conditions. Shape preservation is always desirable in meteorology,, designing car bodies, surgery, designing pipe systems in chemical plants, sectional drawing, engineering, visualization, geometric modeling, etc. There are three basic elements in shape preservation, i.e.,

- Positivity preservation

- Monotonicity preservation

- Convexity preservation

Convexity is an important shape preserving property and its applications are in approximation theory, parameter estimation, optimization theory, engineering design, nonlinear programming, designing telecommunication system, etc. In addition, convergence, smoothness, error estimation, approximation order and stability, convexity preservation is also very emerging and hot topic in graphing smooth limiting curves and surface designs. In the past couple of years, numerous research articles have been published on this topic. Generally, convexity preservation is basic in geometry but also widely used in other branches of mathematics like calculus of variation, partial differential equations, probability theory, graph theory, complex analysis, coding theory, functional analysis and discrete mathematics etc. Convexity preservation also has crucial role in other branches of sciences such as economics, physics, chemistry and biology etc.

\section{Literature review}

The Non-stationary subdivision schemes were established for the first time by Dyn and Levin [3] in which they proposed binary stationary and non-stationary subdivision schemes in 1992. In 2002, Jena et al. [4] presented a subdivision algorithm for trigonometric spline curves. Later in 2003 Jena et al. [5] also proposed a binary 4-point interpolating non-stationary subdivision scheme which can generate $C^{1}$ limit curve. In 2007, Beccari et al. [6, 7] proposed an interpolating 4-point $C^{2}$ ternary non-stationary subdivision scheme with tension control and a non-stationary uniform tension controlled interpolating 4-point scheme reproducing conics respectively. A reasonable amount of research on the topic of non-stationary subdivision schemes has been published in the past years. Recent proposals of non-stationary subdivision schemes Daniel and Shunmugaraj [8, 9, 10], Conti and Romani [11, 12], Siddiqi et al. [13, 14], Bari and Mustafa [15] and Tan et al. [16] have introduced new interesting artifacts in the subdivision museum.

A lot of research papers have been publicized on shape preservation in the past couple of years. In 1994, Méhauté and Utreras [17] introduced a method to overcome the problem of shape preservation in interpolating subdivision. In 1998, Kuijt and Damme [18] construct local subdivision schemes that interpolate functional univariate data that preserve convexity. Dyn etal. [19] examine the convexity preservation properties of 4-point binary interpolating subdivision scheme of Dyn et al. [20] in 1999. In 2009, Cai [21] discuss the convexity preservation of 4-point 
ternary stationary subdivision scheme. Recently, in 2017, Wang and Li [22] proposed a family of convexity preserving subdivision schemes and Akram et al. [23] deduced the shape preserving properties of binary 4-point non-stationary interpolating subdivision schemes.

The paper is organized as follows: In Section 2, we recall ternary 4-point non-stationary interpolating subdivision scheme [1]. In Section 3, 4 and 5, we deduce positivity, monotonicity and convexity preservation respectively. The applications and properties i.e. shape of limit curve and curvature are compared in Section 6. The closing Section 7 is devoted for conclusion.

\section{The ternary 4-point non-stationary scheme}

The following ternary 4-point non-stationary interpolating subdivision scheme constructed by Beccari et al. [1] have been considered:

Definition 2.1. Given a set of initial control points $P^{0}=\left\{\left(i, f_{i}^{0}\right) \in \mathbb{R}^{d}\right\}_{i=-2}^{n+2}$ and the set of control points at $k^{\text {th }}$ subdivision step by $P^{k}=\left\{\left(i, f_{i}^{k}\right) \in \mathbb{R}^{d}\right\}_{2^{k} n+2}^{n+2},(k \geq 0, k \in \mathbb{Z})$. Then the refined reproducing curves at $(k+1)^{\text {th }}$ subdivision step are obtained by applying following subdivision rules:

$$
\left\{\begin{array}{l}
f_{3 i}^{k+1}=f_{i}^{k} \\
f_{3 i+1}^{k+1}=a_{0} f_{i-1}^{k}+a_{1} f_{i}^{k}+a_{2} f_{i+1}^{k}+a_{3} f_{i+2}^{k} \\
f_{3 i+2}^{k+1}=a_{3} f_{i-1}^{k}+a_{2} f_{i}^{k}+a_{1} f_{i+1}^{k}+a_{0} f_{i+2}^{k}
\end{array}\right.
$$

with

$$
\begin{aligned}
& a_{0}=\frac{2 \omega_{k+1}+1}{3\left(1-\omega_{k+1}\right)\left(1+\omega_{k+1}\right)^{3}}, \\
& a_{1}=\frac{\left(1-2\left(\omega_{k+1}\right)^{3}\right)\left(2+\omega_{k+1}\right)}{3\left(1-\omega_{k+1}\right)\left(1+\omega_{k+1}\right)^{3}} \\
& a_{2}=\frac{2\left(-1+\omega_{k+1}\right)-\left(\omega_{k+1}\right)^{3}\left(2+\omega_{k+1}\right)}{3\left(1-\omega_{k+1}\right)\left(1+\omega_{k+1}\right)^{3}}, \\
& a_{3}=\frac{\omega_{k+1}+2}{3\left(1-\omega_{k+1}\right)\left(1+\omega_{k+1}\right)^{3}} .
\end{aligned}
$$

Where the parameter $\omega_{k+1}$ satisfies the recurrence relation

$$
\omega_{k+1}=\Re\left[\left(\frac{\omega_{k}+\sqrt{\left(\omega_{k}\right)^{2}-4}}{2}\right)^{\frac{1}{3}}+\left(\frac{\omega_{k}+\sqrt{\left(\omega_{k}\right)^{2}-4}}{2}\right)^{-\frac{1}{3}}\right] .
$$

The subdivision scheme (1) generates $C^{1}$ continuous limit curves. In particular, for the sequence defined by (2) with $\omega_{0} \in(-2,+\infty)$, it holds respectively: 
- if $-2<\omega_{0}<2$, then $\omega_{0}=2 \cos (s)$ for some $s \in(0, \pi)$, and the scheme reproduces the trigonometric functions;

- if $\omega_{0}=2$, then the scheme reproduces the cubic polynomials;

- if $\omega_{0} \in(2, \infty)$, then $\omega_{0}=2 \cosh (s)$ for some $s>0$, and the scheme reproduces the hyperbolic functions.

In order to determine the shape preseving conditions we will consider whenever subdivision scheme (1) exact for the hyperbolic functions $\left(\omega_{0}>2\right)$ and $\omega_{k+1} \in(2, \infty)$ at each subdivision step for this choice of the initial tension parameter $\omega_{0}$.

\section{Positivity preservation}

To derive the positivity preserving condition for a arbitrary finite number of $\mathrm{n}$ subdivision steps, Proposition 1 is given below.

Proposition 1. Taking, $u_{i}^{n}=\frac{f_{i+1}^{n}}{f_{i}^{n}}$ and $U^{n}=\max _{i}\left\{u_{i}^{n}, \frac{1}{u_{i}^{n}}\right\}, n \in \mathbb{Z}, n>0$. Further suppose that for any $n \in \mathbb{Z}_{+}$, the initial control points are positive, i.e., $f_{i}^{0}>0, i \in \mathbb{Z}$, such that

$$
U^{0}<\frac{2\left(\omega_{n+1}\right)^{4}+4\left(\omega_{n+1}\right)^{3}-4 \omega_{n+1}-5}{2 \omega_{n+1}+1}=\alpha_{n}
$$

then $U^{n}<\alpha_{n}, f_{i}^{n}>0,\left(n \in \mathbb{Z}_{+}, i \in \mathbb{Z}\right)$, that is, the control points generated by the subdivision scheme (1) at $n^{\text {th }}$ subdivision step are also positive.

Proof. In order to prove Proposition 1 we use mathematical induction on $\mathrm{n}$. As $\omega_{k+1} \in(2, \infty)$, $\forall k \in \mathbb{Z}_{+}$, so it automatically gives

$$
\alpha_{k}=\frac{2\left(\omega_{k+1}\right)^{4}+4\left(\omega_{k+1}\right)^{3}-4 \omega_{k+1}-5}{2 \omega_{k+1}+1}>2
$$

(i). Obviously, the statement holds for $n=0$, i.e., $f_{i}^{0}>0, U^{0}<\alpha_{n}, i \in \mathbb{Z}$.

(ii). Suppose that $f_{i}^{0}>0$ and $U^{n}<\alpha_{n}, i \in \mathbb{Z}$ holds for some $n \in \mathbb{Z}_{+}$, then it will be proved that $f_{i}^{n+1}>0$ and $U^{n+1}<\alpha_{n}$. Obviously, $\frac{1}{\alpha_{n}}<u_{i}^{n}<\alpha_{n}$ and $\frac{1}{\alpha_{n}}<\frac{1}{u_{i}^{n}}<\alpha_{n}$.

From definition of subdivision scheme (1),

$$
f_{3 i}^{n+1}>0
$$


and

$$
\begin{aligned}
f_{3 i+1}^{n+1} & =a_{0} f_{i-1}^{n}+a_{1} f_{i}^{n}+a_{2} f_{i+1}^{n}+a_{3} f_{i+2}^{n} \\
& =f_{i}^{n}\left[a_{0} \frac{1}{u_{i-1}^{n}}+a_{1}+a_{2} u_{i}^{n}+a_{3} u_{i}^{n} u_{i+1}^{n}\right] \\
& >f_{i}^{n}\left[a_{0} \alpha_{n}+a_{1}+\left(a_{2}+a_{3} \alpha_{n}\right) u_{i}^{n}\right] \\
& >f_{i}^{n}\left[a_{0} \alpha_{n}+a_{1}+\left(a_{2}+a_{3} \alpha_{n}\right) \frac{1}{\alpha_{n}}\right] \\
& =\frac{f_{i}^{n}}{\alpha_{n}}\left[a_{0} \alpha_{n}^{2}+\left(a_{1}+a_{3}\right) \alpha_{n}+a_{2}\right] \\
& =\frac{f_{i}^{n}}{3 \alpha_{n}\left(1-\omega_{n+1}\right)\left(1+\omega_{n+1}\right)^{3}}\left[\left(2 \omega_{n+1}+1\right) \alpha_{n}^{2}-\left\{2 \omega_{n+1}^{4}+4 \omega_{n+1}^{3}-2 \omega_{n+1}-4\right\} \alpha_{n}\right. \\
& \left.-\left\{\omega_{n+1}^{4}+2 \omega_{n+1}^{3}-2 \omega_{n+1}+2\right\}\right] \\
& =\frac{f_{i}^{n}}{3 \alpha_{n}\left(1-\omega_{n+1}\right)\left(1+\omega_{n+1}\right)^{3}}\left[3\left(1-\omega_{n+1}\right)\left(1+\omega_{n+1}\right)^{3}\right] \\
& =\frac{f_{i}^{n}}{\alpha_{n}}>0 .
\end{aligned}
$$

Similarly

$$
\begin{aligned}
f_{3 i+2}^{n+1} & =a_{3} f_{i-1}^{n}+a_{2} f_{i}^{n}+a_{1} f_{i+1}^{n}+a_{0} f_{i+2}^{n} \\
& =f_{i}^{n}\left[a_{3} \frac{1}{u_{i-1}^{n}}+a_{2}+a_{1} u_{i}^{n}+a_{0} u_{i}^{n} u_{i+1}^{n}\right] \\
& >f_{i}^{n}\left[a_{3} \alpha_{n}+a_{2}+\left(a_{1}+a_{0} \alpha_{n}\right) u_{i}^{n}\right] \\
& >f_{i}^{n}\left[a_{3} \alpha_{n}+a_{2}+\left(a_{1}+a_{0} \alpha_{n}\right) \frac{1}{\alpha_{n}}\right] \\
& =\frac{f_{i}^{n}}{\alpha_{n}}\left[a_{3} \alpha_{n}^{2}+\left(a_{2}+a_{0}\right) \alpha_{n}+a_{1}\right] \\
& =\frac{f_{i}^{n}}{3 \alpha_{n}\left(1-\omega_{n+1}\right)\left(1+\omega_{n+1}\right)^{3}}\left[\left(\omega_{n+1}+2\right) \alpha_{n}^{2}-\left\{\omega_{n+1}^{4}+2 \omega_{n+1}^{3}-4 \omega_{n+1}+1\right\} \alpha_{n}\right. \\
& \left.-\left\{2 \omega_{n+1}^{4}+4 \omega_{n+1}^{3}-\omega_{n+1}-2\right\}\right] \\
& =\frac{f_{i}^{n}}{\alpha_{n}}>0
\end{aligned}
$$

Combining Equations (4), (5) and (6), we get $f_{i}^{n+1}>0$. 
(iii). Here we prove $U^{n+1}<\alpha_{n}$, it is shown that $u_{i}^{n+1}<\alpha_{n}$ and $\frac{1}{u_{i}^{n+1}}<\alpha_{n}$. As

$$
\begin{aligned}
u_{3 i}^{n+1} & =\frac{f_{3 i+1}^{n+1}}{f_{3 i}^{n+1}} \\
& =\frac{a_{0} f_{i-1}^{n}+a_{1} f_{i}^{n}+a_{2} f_{i+1}^{n}+a_{3} f_{i+2}^{n}}{f_{i}^{n}} \\
& =\frac{f_{i}^{n}\left[a_{0} \frac{1}{u_{i-1}^{n}}+a_{1}+a_{2} u_{i}^{n}+a_{3} u_{i}^{n} u_{i+1}^{n}\right]}{f_{i}^{n}} \\
& =a_{0} \frac{1}{u_{i-1}^{n}}+a_{1}+a_{2} u_{i}^{n}+a_{3} u_{i}^{n} u_{i+1}^{n}
\end{aligned}
$$

Thus

$$
\begin{aligned}
u_{3 i}^{n+1}-\alpha_{n} & =a_{0} \frac{1}{u_{i-1}^{n}}+a_{1}+a_{2} u_{i}^{n}+a_{3} u_{i}^{n} u_{i+1}^{n}-\alpha_{n} \\
& <a_{0} \frac{1}{\alpha_{n}}+a_{1}+\left(a_{2}+a_{3} \frac{1}{\alpha_{n}}\right) u_{i}^{n}-\alpha_{n} \\
& <a_{0} \frac{1}{\alpha_{n}}+a_{1}+\left(a_{2}+a_{3} \frac{1}{\alpha_{n}}\right) \alpha_{n}-\alpha_{n} \\
& =a_{0} \frac{1}{\alpha_{n}}+a_{1}+a_{2} \alpha_{n}+a_{3}-\alpha_{n} \\
& =\frac{1}{\alpha_{n}}\left[a_{0}+\left(a_{1}+a_{3}\right) \alpha_{n}+\left(a_{2}-1\right) \alpha_{n}^{2}\right] \\
& =\frac{1}{3 \alpha_{n}\left(1-\omega_{n+1}\right)\left(1+\omega_{n+1}\right)^{3}}\left[\left(2 \omega_{n+1}^{4}+4 \omega_{n+1}^{3}-4 \omega_{n+1}-5\right) \alpha_{n}^{2}\right. \\
& \left.+\left(-2 \omega_{n+1}^{4}-4 \omega_{n+1}^{3}+2 \omega_{n+1}+4\right) \alpha_{n}+\left(2 \omega_{n+1}+1\right)\right] \\
= & \frac{\left(\alpha_{n}-1\right)}{3 \alpha_{n}\left(1-\omega_{n+1}\right)\left(1+\omega_{n+1}\right)^{3}}\left[\left(2 \omega_{n+1}^{4}+4 \omega_{n+1}^{3}-4 \omega_{n+1}-5\right) \alpha_{n}-\left(2 \omega_{n+1}+1\right)\right] \\
= & \frac{\left(\alpha_{n}-1\right)^{2}\left(2 \omega_{n+1}+1\right)\left(\alpha_{n}+1\right)}{3 \alpha_{n}\left(1-\omega_{n+1}\right)\left(1+\omega_{n+1}\right)^{3}} \\
< & 0 .
\end{aligned}
$$

Using the fact that $\alpha_{n}>2$ and $\omega_{n+1} \in(2, \infty)$, so we have,

$$
u_{3 i}^{n+1}<\alpha_{n}
$$

and

$$
\begin{aligned}
u_{3 i+1}^{n+1} & =\frac{f_{3 i+2}^{n+1}}{f_{3 i+1}^{n+1}}=\frac{a_{3} f_{i-1}^{n}+a_{2} f_{i}^{n}+a_{1} f_{i+1}^{n}+a_{0} f_{i+2}^{n}}{a_{0} f_{i-1}^{n}+a_{1} f_{i}^{n}+a_{2} f_{i+1}^{n}+a_{3} f_{i+2}^{n}} \\
& =\frac{f_{i}^{n}\left[a_{3} \frac{1}{u_{i-1}^{n}}+a_{2}+a_{1} u_{i}^{n}+a_{0} u_{i}^{n} u_{i+1}^{n}\right]}{f_{i}^{n}\left[a_{0} \frac{1}{u_{i-1}^{n}}+a_{1}+a_{2} u_{i}^{n}+a_{3} u_{i}^{n} u_{i+1}^{n}\right]}
\end{aligned}
$$


thus

$$
\begin{aligned}
u_{3 i+1}^{n+1} & =\frac{a_{3} \frac{1}{u_{i-1}^{n}}+a_{2}+a_{1} u_{i}^{n}+a_{0} u_{i}^{n} u_{i+1}^{n}-\alpha_{n}\left[a_{0} \frac{1}{u_{i-1}^{n}}+a_{1}+a_{2} u_{i}^{n}+a_{3} u_{i}^{n} u_{i+1}^{n}\right]}{a_{0} \frac{1}{u_{i-1}^{n}}+a_{1}+a_{2} u_{i}^{n}+a_{3} u_{i}^{n} u_{i+1}^{n}} \\
& =\frac{N}{D} .
\end{aligned}
$$

As in Eq. (5) Denominator $=D>0$ and Numerator $=\mathrm{N}$, satisfies

$$
\begin{aligned}
N & =a_{3} \frac{1}{u_{i-1}^{n}}+a_{2}+a_{1} u_{i}^{n}+a_{0} u_{i}^{n} u_{i+1}^{n}-\alpha_{n}\left[a_{0} \frac{1}{u_{i-1}^{n}}+a_{1}+a_{2} u_{i}^{n}+a_{3} u_{i}^{n} u_{i+1}^{n}\right] \\
& <a_{3} \frac{1}{\alpha_{n}}+a_{2}+a_{1} u_{i}^{n}+a_{0} u_{i}^{n} u_{i+1}^{n}-\alpha_{n}\left[a_{0} \frac{1}{\alpha_{n}}+a_{1}+a_{2} u_{i}^{n}+a_{3} u_{i}^{n} u_{i+1}^{n}\right] \\
& <a_{3} \frac{1}{\alpha_{n}}+a_{2}+\left(a_{1}+a_{0} \frac{1}{\alpha_{n}}\right) u_{i}^{n}-\alpha_{n}\left[a_{0} \frac{1}{\alpha_{n}}+a_{1}+\left(a_{2}+a_{3} \frac{1}{\alpha_{n}}\right) u_{i}^{n}\right] \\
& <a_{3} \frac{1}{\alpha_{n}}+a_{2}+\left(a_{1}+a_{0} \frac{1}{\alpha_{n}}\right) u_{i}^{n}-\alpha_{n}\left[a_{0} \frac{1}{\alpha_{n}}+a_{1}+\left(a_{2}+a_{3} \frac{1}{\alpha_{n}}\right) u_{i}^{n}\right] \\
& <a_{3} \frac{1}{\alpha_{n}}+a_{2}+\left(a_{1}+a_{0} \frac{1}{\alpha_{n}}\right) \alpha_{n}-\alpha_{n}\left[a_{0} \frac{1}{\alpha_{n}}+a_{1}+\left(a_{2}+a_{3} \frac{1}{\alpha_{n}}\right) \alpha_{n}\right] \\
& =a_{3} \frac{1}{\alpha_{n}}+a_{2}-a_{2} \alpha_{n}^{2}-a_{3} \alpha_{n} \\
& =-\frac{1}{\alpha_{n}}\left[a_{2} \alpha_{n}^{3}+a_{3} \alpha_{n}^{2}-a_{2} \alpha_{n}-a_{3}\right] \\
& =-\frac{1}{\alpha_{n}}\left[\alpha_{n}^{2}-1\right]\left[a_{2} \alpha_{n}+a_{3}\right] \\
& <0 .
\end{aligned}
$$

Using the fact that $\alpha_{n}>2$ and $\omega_{n+1} \in(2, \infty)$, so we have,

$$
u_{3 i+1}^{n+1}<\alpha_{n}
$$

Similarly

$$
\begin{aligned}
u_{3 i+2}^{n+1} & =\frac{f_{3 i+3}^{n+1}}{f_{3 i+2}^{n+1}}=\frac{f_{i+1}^{n}}{a_{3} f_{i-1}^{n}+a_{2} f_{i}^{n}+a_{1} f_{i+1}^{n}+a_{0} f_{i+2}^{n}} \\
& =\frac{f_{i+1}^{n}}{f_{i+1}^{n}\left[a_{3} \frac{1}{u_{i-1}^{n} u_{i}^{n}}+a_{2} \frac{1}{u_{i}^{n}}+a_{1}+a_{0} u_{i+1}^{n}\right]}
\end{aligned}
$$

thus

$$
\begin{aligned}
u_{3 i+2}^{n+1}-\alpha_{n} & =\frac{1-\alpha_{n}\left[a_{3} \frac{1}{u_{i-1}^{n} u_{i}^{n}}+a_{2} \frac{1}{u_{i}^{n}}+a_{1}+a_{0} u_{i+1}^{n}\right]}{\left[a_{3} \frac{1}{u_{i-1}^{n} u_{i}^{n}}+a_{2} \frac{1}{u_{i}^{n}}+a_{1}+a_{0} u_{i+1}^{n}\right]} \\
& =\frac{N}{D} .
\end{aligned}
$$


As in Eq. (6) Denominator $=D>0$ and Numerator $=\mathrm{N}$, satisfies

$$
\begin{aligned}
N= & 1-\alpha_{n}\left[a_{3} \frac{1}{u_{i-1}^{n} u_{i}^{n}}+a_{2} \frac{1}{u_{i}^{n}}+a_{1}+a_{0} u_{i+1}^{n}\right] \\
= & 1-\left(a_{3} \frac{1}{u_{i-1}^{n}}+a_{2}\right) \frac{\alpha_{n}}{u_{i}^{n}}-a_{1} \alpha_{n}-a_{0} \alpha_{n} u_{i+1}^{n} \\
< & 1-\left(a_{3} \alpha_{n}+a_{2}\right) \frac{\alpha_{n}}{u_{i}^{n}}-a_{1} \alpha_{n}-a_{0} \alpha_{n}^{2} \\
< & 1-\left(a_{3} \alpha_{n}+a_{2}\right)-a_{1} \alpha_{n}-a_{0} \alpha_{n}^{2} \\
= & -a_{0} \alpha_{n}^{2}-\left(a_{3}+a_{1}\right) \alpha_{n}+1-a_{2} \\
= & -\left\{\frac{2 \omega_{n+1}+1}{3\left(1-\omega_{n+1}\right)\left(1+\omega_{n+1}\right)^{3}}\right\} \alpha_{n}^{2}+2\left\{\frac{\omega_{n+1}^{4}+2 \omega_{n+1}^{3}-\omega_{n+1}-2}{3\left(1-\omega_{n+1}\right)\left(1+\omega_{n+1}\right)^{3}}\right\} \alpha_{n} \\
& -\left\{\frac{2 \omega_{n+1}^{4}+4 \omega_{n}^{3}-4 \omega_{n+1}-5}{3\left(1-\omega_{n+1}\right)\left(1+\omega_{n+1}\right)^{3}}\right\} \\
= & \left(\alpha_{n}-1\right)\left(\alpha_{n}-\frac{2 \omega_{n+1}^{4}+4 \omega_{n+1}^{3}-4 \omega_{n+1}-5}{2 \omega_{n+1}+1}\right) \\
= & 0 .
\end{aligned}
$$

Using the fact that $\alpha_{n}>2$ and $\omega_{n+1} \in(2, \infty)$, so we have,

$$
u_{3 i+2}^{n+1}<\alpha_{n}
$$

Combining Eqs. (7), (8) and (9), we get $u_{i}^{n+1}<\alpha_{n}$.

Similarly, it can be proved that $\frac{1}{u_{i}^{n+1}}<\alpha_{n}$.

As, $U^{n+1}=\max _{i}\left\{u_{i}^{n+1}, \frac{1}{u_{i}^{n+1}}\right\}$, thus $U^{n+1}<\alpha_{n}$.

Hence, by mathematical induction we get $f_{i}^{n}>0, U^{n}<\alpha_{n}$.

Now, we establish the positivity preserving condition in the limiting case, as $n \rightarrow \infty$. As the parameter $\omega_{n+1}$ given in Eq. (2) satisfies

$$
\lim _{n \rightarrow \infty} \omega_{n+1}=2
$$

Consequently, $\lim _{n \rightarrow \infty} \alpha_{n}=\frac{51}{5}$ in Theorem 1 and note that the proof can be followed from Proposition 1.

Theorem 1. Suppose that the initial control points are positive, s.t.

$$
U^{0}<\frac{51}{5}
$$

then the limit curves generated by the subdivision scheme (1) are still positive. 


\section{Monotonicity preservation}

In the following Proposition 2, we first establish monotonicity preserving condition for the finite number of $\mathrm{n}$ subdivision steps.

Denote the first order divided difference by $D_{i}^{k}=2^{k}\left(f_{i+1}^{k}-f_{i}^{k}\right)$. Thus the first order divided difference for the subdivision scheme (1) can written as:

$$
\begin{gathered}
D_{3 i}^{k+1}=\frac{-2\left(2 \omega_{k+1}+1\right) D_{i-1}^{k}-2\left(\omega_{k+1}^{4}+2 \omega_{k+1}^{3}-3 \omega_{k+1}\right) D_{i}^{k}+2\left(\omega_{k+1}+2\right) D_{i+1}^{k}}{3\left(1-\omega_{k+1}\right)\left(1+\omega_{k+1}\right)^{3}} \\
D_{3 i+1}^{k+1}=\frac{2\left(\omega_{k+1}-1\right) D_{i-1}^{k}-2\left(\omega_{k+1}^{4}+2 \omega_{k+1}^{3}-3\right) D_{i}^{k}+2\left(\omega_{k+1}-1\right) D_{i+1}^{k}}{3\left(1-\omega_{k+1}\right)\left(1+\omega_{k+1}\right)^{3}} \\
D_{3 i+2}^{k+1}=\frac{2\left(\omega_{k+1}+2\right) D_{i-1}^{k}-2\left(\omega_{k+1}^{4}+2 \omega_{k+1}^{3}-3 \omega_{k+1}\right) D_{i}^{k}-2\left(2 \omega_{k+1}+1\right) D_{i+1}^{k}}{3\left(1-\omega_{k+1}\right)\left(1+\omega_{k+1}\right)^{3}}
\end{gathered}
$$

Proposition 2. Taking, $v_{i}^{n}=\frac{D_{i+1}^{n}}{D_{i}^{n}}$ and $V^{n}=\max _{i}\left\{v_{i}^{n}, \frac{1}{v_{i}^{n}}\right\}, n \in \mathbb{Z}, n>0$. Further suppose that for any $n \in \mathbb{Z}_{+}$, the initial control points are strictly monotonic increasing, i.e., $D_{i}^{0}>0$, $i \in \mathbb{Z}$, such that

$$
\begin{aligned}
V^{0} & \leq \frac{\omega_{n+1}^{4}+2 \omega_{n+1}^{3}-3 \omega_{n+1}-3}{2 \omega_{n+1}+4} \\
& +\frac{\sqrt{\omega_{n+1}^{8}+4 \omega_{n+1}^{7}+4 \omega_{n+1}^{6}-6 \omega_{n+1}^{5}-18 \omega_{n+1}^{4}-12 \omega_{n+1}^{3}+5 \omega_{n+1}^{2}+14 \omega_{n+1}+17}}{2 \omega_{n+1}+4} \\
& =\beta_{n},
\end{aligned}
$$

then $V^{n}<\beta_{n}, D_{i}^{n}>0,\left(n \in \mathbb{Z}_{+}, i \in \mathbb{Z}\right)$, that is, the control points generated by the subdivision scheme (1) at $n^{\text {th }}$ subdivision step are still strictly monotonic increasing.

Proof. In order to prove Proposition 2, we use mathematical induction on $n$. As $\omega_{k+1} \in(2, \infty)$, so it conveniently gives

$$
\begin{aligned}
\beta_{k} & =\frac{\omega_{k+1}^{4}+2 \omega_{k+1}^{3}-3 \omega_{k+1}-3}{2 \omega_{k+1}+4} \\
& +\frac{\sqrt{\omega_{k+1}^{8}+4 \omega_{k+1}^{7}+4 \omega_{k+1}^{6}-6 \omega_{k+1}^{5}-18 \omega_{k+1}^{4}-12 \omega_{k+1}^{3}+5 \omega_{k+1}^{2}+14 \omega_{k+1}+17}}{2 \omega_{k+1}+4} \\
& >2 .
\end{aligned}
$$

(i). Obviously, the statement holds for $n=0$, i.e., $D_{i}^{0}>0, V^{0}<\beta_{n}, i \in \mathbb{Z}$.

(ii). Suppose that $D_{i}^{0}>0$ and $V^{n} \leq \beta_{n}, i \in \mathbb{Z}$ holds for some $n \in \mathbb{Z}_{+}$, then it will be proved that $D_{i}^{n+1}>0$ and $V^{n+1}<\beta_{n}$. Obviously, $\frac{1}{\beta_{n}}<v_{i}^{n}<\beta_{n}$ and $\frac{1}{\beta_{n}}<\frac{1}{v_{i}^{n}}<\beta_{n}$. 
Using Eq. (10),

$$
\begin{aligned}
D_{3 i}^{k+1} & =\frac{D_{i}^{n}}{3\left(1-\omega_{n+1}\right)\left(1+\omega_{n+1}\right)^{3}}\left[-2\left(2 \omega_{n+1}+1\right) \frac{1}{v_{i-1}^{n}}-2\left(\omega_{n+1}^{4}+2 \omega_{k+1}^{3}-3 \omega_{n+1}\right)+2\left(\omega_{n+1}+2\right) v_{i}^{n}\right] \\
& \geq \frac{D_{i}^{n}}{3\left(1-\omega_{n+1}\right)\left(1+\omega_{n+1}\right)^{3}}\left[-2\left(2 \omega_{n+1}+1\right) \frac{1}{\beta_{n}}-2\left(\omega_{n+1}^{4}+2 \omega_{k+1}^{3}-3 \omega_{n+1}\right)+2\left(\omega_{n+1}+2\right) \beta_{n}\right] \\
& =\frac{D_{i}^{n}}{3 \beta_{n}\left(1-\omega_{n+1}\right)\left(1+\omega_{n+1}\right)^{3}}\left[-2\left(2 \omega_{n+1}+1\right)-2\left(\omega_{n+1}^{4}+2 \omega_{k+1}^{3}-3 \omega_{n+1}\right) \beta_{n}+2\left(\omega_{n+1}+2\right) \beta_{n}^{2}\right] \\
& =\frac{D_{i}^{n}}{3 \beta_{n}\left(1-\omega_{n+1}\right)\left(1+\omega_{n+1}\right)^{3}\left(\omega_{n}+2\right)} \times \\
& {\left[\omega_{n+1}^{4}+2 \omega_{n+1}^{3}+2 \omega_{n+1}^{2}+\omega_{n+1}-3\right.} \\
& \left.+\sqrt{\omega_{n+1}^{8}+4 \omega_{n+1}^{7}+4 \omega_{n+1}^{6}-6 \omega_{n+1}^{5}-18 \omega_{n+1}^{4}-12 \omega_{n+1}^{3}+5 \omega_{n+1}^{2}+14 \omega_{n+1}+17}\right] \\
& >0 .
\end{aligned}
$$

Similarly, from Eqs. (11) and (12), we get

$$
D_{3 i+1}^{n+1}>0, \quad D_{3 i+2}^{n+1}>0
$$

Combining Eqs. (14) and (15), we have $D_{i}^{n+1}>0$.

(iii). Here we prove $V^{n+1}<\beta_{n}$, it is shown that $v_{i}^{n+1}<\beta_{n}$ and $\frac{1}{v_{i}^{n+1}}<\beta_{n}$.

Since,

$$
\begin{aligned}
v_{3 i}^{n+1} & =\frac{D_{3 i+1}^{n+1}}{D_{3 i}^{n+1}} \\
& =\frac{2\left(\omega_{n+1}+1\right) D_{i-1}^{n}-2\left(\omega_{n+1}^{4}+2 \omega_{n+1}^{3}-3\right) D_{i}^{n}+2\left(\omega_{n+1}-1\right) D_{i+1}^{n}}{-2\left(2 \omega_{n+1}+1\right) D_{i-1}^{n}-2\left(\omega_{n+1}^{4}+2 \omega_{n+1}^{3}-3 \omega_{n+1}\right) D_{i}^{n}+2\left(\omega_{n+1}+2\right) D_{i+1}^{n}} \\
& =\frac{2 D_{i}^{n}\left[\left(\omega_{n+1}+1\right) \frac{1}{v_{i-1}^{n}}-\left(\omega_{n+1}^{4}+2 \omega_{n+1}^{3}-3\right)+\left(\omega_{n+1}-1\right) v_{i}^{n}\right]}{2 D_{i}^{n}\left[-\left(2 \omega_{n+1}+1\right) \frac{1}{v_{i-1}^{n}}-\left(\omega_{n+1}^{4}+2 \omega_{n+1}^{3}-3 \omega_{n+1}\right)+\left(\omega_{n+1}+2\right) v_{i}^{n}\right]}
\end{aligned}
$$

thus

$$
\begin{aligned}
v_{3 i}^{n+1}-\beta_{n} & =\frac{1}{\left[-\left(2 \omega_{n+1}+1\right) \frac{1}{v_{i-1}^{n}}-\left(\omega_{n+1}^{4}+2 \omega_{n+1}^{3}-3 \omega_{n+1}\right)+\left(\omega_{n+1}+2\right) v_{i}^{n}\right]} \times \\
& {\left[\left(\omega_{n+1}+1\right) \frac{1}{v_{i-1}^{n}}-\left(\omega_{n+1}^{4}+2 \omega_{n+1}^{3}-3\right)+\left(\omega_{n+1}-1\right) v_{i}^{n}\right] } \\
& -\beta_{n}\left[-\left(2 \omega_{n+1}+1\right) \frac{1}{v_{i-1}^{n}}-\left(\omega_{n+1}^{4}+2 \omega_{n+1}^{3}-3 \omega_{n+1}\right)+\left(\omega_{n+1}+2\right) v_{i}^{n}\right] \\
& =\frac{N}{D} .
\end{aligned}
$$


As in Eq. (14) Denominator $=D>0$ and Numerator $=\mathrm{N}$, satisfies

$$
\begin{aligned}
& N=\left[\left(\omega_{n+1}+1\right) \frac{1}{v_{i-1}^{n}}-\left(\omega_{n+1}^{4}+2 \omega_{n+1}^{3}-3\right)+\left(\omega_{n+1}-1\right) v_{i}^{n}\right] \\
& -\beta_{n}\left[-\left(2 \omega_{n+1}+1\right) \frac{1}{v_{i-1}^{n}}-\left(\omega_{n+1}^{4}+2 \omega_{n+1}^{3}-3 \omega_{n+1}\right)+\left(\omega_{n+1}+2\right) v_{i}^{n}\right] \\
& =\left[\left(\omega_{n+1}-1\right)+\left(2 \omega_{n+1}+1\right)\right] \frac{1}{v_{i-1}^{n}} \\
& +\left[-\left(\omega_{n+1}^{4}+2 \omega_{n+1}^{3}-3\right)+\left(\omega_{n+1}^{4}+2 \omega_{n+1}^{3}-\omega_{n+1}\right) \beta_{n}\right]+\left[\left(\omega_{n+1}-1\right)+\left(\omega_{n+1}+2\right) \beta_{n}\right] v_{i}^{n} \\
& <\left[\left(\omega_{n+1}-1\right)+\left(2 \omega_{n+1}+1\right)\right] \frac{1}{\beta_{n}} \\
& +\left[-\left(\omega_{n+1}^{4}+2 \omega_{n+1}^{3}-3\right)+\left(\omega_{n+1}^{4}+2 \omega_{n+1}^{3}-\omega_{n+1}\right) \beta_{n}\right]+\left[\left(\omega_{n+1}-1\right)+\left(\omega_{n+1}+2\right) \beta_{n}\right] \beta_{n} \\
& =-\frac{1}{\beta_{n}}\left[\left(\omega_{n+1}+2\right) \beta_{n}^{3}-\left(\omega_{n+1}^{4}+2 \omega_{n+1}^{3}-2 \omega_{n+1}-1\right) \beta_{n}^{2}\right. \\
& \left.-\left(-\omega_{n+1}^{4}-2 \omega_{n+1}^{3}+2 \omega_{n+1}+4\right) \beta_{n}-\left(\omega_{n+1}+1\right)\right] \\
& =-\frac{1}{\beta_{n}}\left[\beta_{n}-1\right]\left[\left(\omega_{n+1}+2\right) \beta_{n}^{2}+\left(-\omega_{n+1}^{4}-2 \omega_{n+1}^{3}+3 \omega_{n+1}+3\right) \beta_{n}+\left(\omega_{n+1}-1\right)\right] \\
& =-\frac{1}{\beta_{n}}\left[\beta_{n}-1\right] \times \\
& {\left[\beta_{n}-\frac{\omega_{n+1}^{4}+2 \omega_{n+1}^{3}-3 \omega_{n+1}-3}{2 \omega_{n+1}+4}\right.} \\
& \left.+\frac{\sqrt{\omega_{n+1}^{8}+4 \omega_{n+1}^{7}+4 \omega_{n+1}^{6}-6 \omega_{n+1}^{5}-18 \omega_{n+1}^{4}-12 \omega_{n+1}^{3}+5 \omega_{n+1}^{2}+14 \omega_{n+1}+17}}{2 \omega_{n+1}+4}\right] \\
& {\left[\beta_{n}-\frac{\omega_{n+1}^{4}+2 \omega_{n+1}^{3}-3 \omega_{n+1}-3}{2 \omega_{n+1}+4}\right.} \\
& \left.-\frac{\sqrt{\omega_{n+1}^{8}+4 \omega_{n+1}^{7}+4 \omega_{n+1}^{6}-6 \omega_{n+1}^{5}-18 \omega_{n+1}^{4}-12 \omega_{n+1}^{3}+5 \omega_{n+1}^{2}+14 \omega_{n+1}+17}}{2 \omega_{n+1}+4}\right] \\
& \leq 0 \text {. }
\end{aligned}
$$

Hence,

$$
v_{3 i}^{n+1} \leq \beta_{n}
$$

Similarly, one can easily show that

$$
v_{3 i+1}^{n+1} \leq \beta_{n}, \quad v_{3 i+2}^{n+1} \leq \beta_{n} .
$$

Combining Eqs. (16) and (17), we have $v_{i}^{n+1}<\beta_{n}$. Applying same method, it can be proved that $\frac{1}{v_{i}^{n+1}} \leq \beta_{n}$ by verifying $\frac{1}{v_{3 i}^{n+1}} \leq \beta_{n}, \frac{1}{v_{3 i+1}^{n+1}} \leq \beta_{n}$ and $\frac{1}{v_{3 i+2}^{n+1}} \leq \beta_{n}$.

As, $V^{n+1}=\max _{i}\left\{v_{i}^{n+1}, \frac{1}{v_{i}^{n+1}}\right\}$, thus $V^{n+1}<\beta_{n}$.

Hence, by mathematical induction we get $D_{i}^{n}>0, V^{n}<\beta_{n}$. 
Now, we establish the monotonicity preserving condition in the limiting case, as $n \rightarrow \infty$. As the parameter $\omega_{n+1}$ given in Eq. (2) satisfies $\lim _{n \rightarrow \infty} \omega_{n+1}=2$. Consequently, $\lim _{n \rightarrow \infty} \beta_{n}=\frac{23}{8}+\frac{1}{8} \sqrt{513}$ in Theorem 2 and note that the proof can be followed from Proposition 2.

Theorem 2. Suppose that the initial control points are strictly monotonic increasing or decreasing, such that,

$$
V^{0} \leq \frac{23}{8}+\frac{1}{8} \sqrt{513}
$$

then the limit curves generated by the subdivision scheme (1) are strictly monotonic increasing or decreasing.

\section{Convexity preservation}

In the following Proposition 3, we first establish convexity preserving condition for the finite number of $n$ subdivision steps.

Denote the second order divided difference by $d_{i}^{k}=2^{2 k-1}\left(f_{i-1}^{k}-2 f_{i}^{k}+f_{i+1}^{k}\right)$. Thus the second order divided difference for the subdivision scheme (1) can written as:

$$
\begin{aligned}
d_{3 i}^{k+1} & =\frac{3}{\left(1-\omega_{n+1}\right)\left(1+\omega_{n+1}\right)^{3}}\left[\left(\omega_{n+1}+2\right) d_{i-1}^{k}-\left(\omega_{n+1}^{4}+2 \omega_{n+1}^{3}-6 \omega_{n+1}-3\right) d_{i}^{k}\right. \\
& \left.+\left(\omega_{n+1}+2\right) d_{i+1}^{k}\right], \\
d_{3 i+1}^{k+1} & =\frac{-9}{\left(1-\omega_{n+1}\right)\left(1+\omega_{n+1}\right)^{3}}\left[\omega_{n+1} d_{i}^{k}+d_{i+1}^{k}\right], \\
d_{3 i+2}^{k+1} & =\frac{-9}{\left(1-\omega_{n+1}\right)\left(1+\omega_{n+1}\right)^{3}}\left[d_{i}^{k}+\omega_{n+1} d_{i+1}^{k}\right] .
\end{aligned}
$$

Proposition 3. Suppose, $r_{i}^{n}=\frac{d_{i+1}^{n}}{d_{i}^{n}}$ and $R^{n}=\max _{i}\left\{r_{i}^{n}, \frac{1}{n_{i}^{n}}\right\}, n \in \mathbb{Z}, n>0$. Further suppose that for any $n \in \mathbb{Z}_{+}$, the initial control points are strictly convex, i.e., $d_{i}^{0}>0, i \in \mathbb{Z}$, such that

$$
\begin{aligned}
R^{0} & \leq \frac{\omega_{n+1}^{4}+2 \omega_{n+1}^{3}-6 \omega_{n+1}-6+\sqrt{\omega_{n+1}^{8}+4 \omega_{n+1}^{7}+4 \omega_{n+1}^{6}-12 \omega_{n+1}^{5}-36 \omega_{n+1}^{4}-24 \omega_{n+1}^{3}+60 \omega_{n+1}^{2}+120 \omega_{n+1}+36}}{4\left(\omega_{n+1}+2\right)} \\
& =\gamma_{n}
\end{aligned}
$$

then $R^{n}<\gamma_{n}, d_{i}^{n}>0,\left(n \in \mathbb{Z}_{+}, i \in \mathbb{Z}\right)$, that is, the control points generated by the subdivision scheme (1) at $n^{\text {th }}$ subdivision step are still strictly convex.

Proof. In order to prove Proposition 3, we use mathematical induction on n. As $\omega_{k+1} \in(2, \infty)$, so it automatically gives

$$
\begin{aligned}
\gamma_{k} & =\frac{\omega_{k+1}^{4}+2 \omega_{k+1}^{3}-6 \omega_{k+1}-6+\sqrt{\omega_{k+1}^{8}+4 \omega_{k+1}^{7}+4 \omega_{k+1}^{6}-12 \omega_{k+1}^{5}-36 \omega_{k+1}^{4}-24 \omega_{k+1}^{3}+60 \omega_{k+1}^{2}+120 \omega_{k+1}+36}}{4\left(\omega_{k+1}+2\right)} \\
& >2 .
\end{aligned}
$$

(i). Obviously, the statement holds for $n=0$, i.e., $d_{i}^{0}>0, R^{0}<\gamma_{n}, i \in \mathbb{Z}$. 
(ii). Suppose that $d_{i}^{n}>0$ and $R^{n} \leq \gamma_{n}, i \in \mathbb{Z}$ holds for some $n \in \mathbb{Z}_{+}$, then it will be proved that $d_{i}^{n+1}>0$ and $R^{n+1}<\gamma_{n}$. Obviously, $\frac{1}{\gamma_{n}}<r_{i}^{n}<\gamma_{n}$ and $\frac{1}{\gamma_{n}}<\frac{1}{r_{i}^{n}}<\gamma_{n}$.

Using Equation (18) we have,

$$
\begin{aligned}
d_{3 i}^{n+1} & =\frac{3}{\left(1-\omega_{n+1}\right)\left(1+\omega_{n+1}\right)^{3}}\left[\left(\omega_{n+1}+2\right) d_{i-1}^{n}-\left(\omega_{n+1}^{4}+2 \omega_{n+1}^{3}-6 \omega_{n+1}-3\right) d_{i}^{n}+\left(\omega_{n+1}+2\right) d_{i+1}^{n}\right], \\
& =\frac{3 d_{i}^{n}}{\left(1-\omega_{n+1}\right)\left(1+\omega_{n+1}\right)^{3}}\left[\left(\omega_{n+1}+2\right) \frac{1}{r_{i-1}^{n}}-\left(\omega_{n+1}^{4}+2 \omega_{n+1}^{3}-6 \omega_{n+1}-3\right)+\left(\omega_{n+1}+2\right) r_{i}^{n}\right], \\
& \geq \frac{3 d_{i}^{n}}{\left(1-\omega_{n+1}\right)\left(1+\omega_{n+1}\right)^{3}}\left[2 \gamma_{n}\left(\omega_{n+1}+2\right)-\left(\omega_{n+1}^{4}+2 \omega_{n+1}^{3}-6 \omega_{n+1}-3\right)\right], \\
d_{3 i}^{n+1} & >0 .
\end{aligned}
$$

$\because \quad \omega_{n+1} \in(2, \infty)$, thus following inequalities must satisfy, i.e.,

$$
d_{3 i}^{n+1}>0, \quad d_{3 i+1}^{n+1}>0 \quad \text { and } \quad d_{3 i+2}^{n+1}>0 .
$$

$\Longrightarrow d_{i}^{n+1}>0$

(iii). Here we prove $R^{n+1}<\gamma_{n}$, it is shown that $r_{i}^{n+1}<\gamma_{n}$ and $\frac{1}{r_{i}^{n+1}}<\gamma_{n}$.

Since,

$$
\begin{aligned}
r_{3 i}^{n+1} & =\frac{d_{3 i+1}^{n+1}}{d_{3 i}^{n+1}} \\
& =\frac{-3\left[\omega_{n+1} d_{i}^{n}+d_{i+1}^{n}\right]}{\left[\left(\omega_{n+1}+2\right) d_{i-1}^{n}-\left(\omega_{n+1}^{4}+2 \omega_{n+1}^{3}-6 \omega_{n+1}-3\right) d_{i}^{n}+\left(\omega_{n+1}+2\right) d_{i+1}^{n}\right]} \\
& =\frac{-3 d_{i}^{n}\left[\omega_{n+1}+r_{i}^{n}\right]}{d_{i}^{n}\left[\left(\omega_{n+1}+2\right) \frac{1}{r_{i-1}^{n}}-\left(\omega_{n+1}^{4}+2 \omega_{n+1}^{3}-6 \omega_{n+1}-3\right)+\left(\omega_{n+1}+2\right) r_{i}^{n}\right]} \\
r_{31}^{n+1}-\gamma & =\frac{-3\left[\omega_{n+1}+r_{i}^{n}\right]-\gamma\left[\left(\omega_{n+1}+2\right) \frac{1}{r_{i-1}^{n}}-\left(\omega_{n+1}^{4}+2 \omega_{n+1}^{3}-6 \omega_{n+1}-3\right)+\left(\omega_{n+1}+2\right) r_{i}^{n}\right]}{\left[\left(\omega_{n+1}+2\right) \frac{1}{r_{i-1}^{n}}-\left(\omega_{n+1}^{4}+2 \omega_{n+1}^{3}-6 \omega_{n+1}-3\right)+\left(\omega_{n+1}+2\right) r_{i}^{n}\right]} \\
& =\frac{N}{D} . \quad
\end{aligned}
$$


As Denominator $<0$, then $\mathrm{N}$ must satisfies

$$
\begin{aligned}
N & =-3\left[\omega_{n+1}+r_{i}^{n}\right]-\gamma_{n}\left[\left(\omega_{n+1}+2\right) \frac{1}{r_{i-1}^{n}}-\left(\omega_{n+1}^{4}+2 \omega_{n+1}^{3}-6 \omega_{n+1}-3\right)+\left(\omega_{n+1}+2\right) r_{i}^{n}\right] \\
& =-3 \omega_{n+1}+\left(\omega_{n+1}^{4}+2 \omega_{n+1}^{3}-6 \omega_{n+1}-3\right) \gamma_{n}+\left[-3-\left(\omega_{n+1}+2\right) \gamma_{n}\right] r_{i}^{n}-\left(\omega_{n+1}+2\right) \frac{\gamma_{n}}{r_{i-1}^{n}} \\
& \leq-3 \omega_{n+1}+\left(\omega_{n+1}^{4}+2 \omega_{n+1}^{3}-6 \omega_{n+1}-3\right) \gamma_{n}+\left[-3-\left(\omega_{n+1}+2\right) \gamma_{n}\right] \gamma_{n}-\left(\omega_{n+1}+2\right) \gamma_{n}^{2} \\
& =-\left[2\left(\omega_{n+1}+2\right) \gamma_{n}^{2}-\left(\omega_{n+1}^{4}+2 \omega_{n+1}^{3}-6 \omega_{n+1}-6\right) \gamma_{n}+3 \omega_{n+1}\right] \\
& =-\left[\gamma_{n}-\left(\frac{\omega_{n+1}^{4}+2 \omega_{n+1}^{3}-6 \omega_{n+1}-6}{4\left(\omega_{n+1}+2\right)}\right)\right] \\
& \left.\left.+\frac{\sqrt{\omega_{n+1}^{8}+4 \omega_{n+1}^{7}+4 \omega_{n+1}^{6}-12 \omega_{n+1}^{5}-36 \omega_{n+1}^{4}-24 \omega_{n+1}^{3}+60 \omega_{n+1}^{2}+120 \omega_{n+1}+36}}{4\left(\omega_{n+1}+2\right)}\right)\right] \times \\
& {\left[\gamma_{n}-\left(\frac{\omega_{n+1}^{4}+2 \omega_{n+1}^{3}-6 \omega_{n+1}-6}{4\left(\omega_{n+1}+2\right)}\right.\right.} \\
& =0 . \\
& =r_{3 i}^{n+1} \leq \gamma_{n}^{8} .
\end{aligned}
$$

Since,

$$
r_{3 i+1}^{n+1}=\frac{d_{3 i+2}^{n+1}}{d_{3 i+1}^{n+1}}=\frac{d_{i}^{n}\left(1+\omega_{n+1} r_{i}^{n}\right)}{d_{i}^{n}\left(\omega_{n+1}+r_{i}^{n}\right)}
$$

thus

$$
r_{3 i+1}^{n+1}-\gamma_{n}=\frac{\left(1+\omega_{n+1} r_{i}^{n}\right)-\gamma_{n}\left(\omega_{n+1}+r_{i}^{n}\right)}{\left(\omega_{n+1}+r_{i}^{n}\right)}=\frac{N}{D} .
$$

As Denominator $<0$, then $\mathrm{N}$ must satisfies

$$
\begin{aligned}
N & =1+\omega_{n+1} r_{i}^{n}-\omega_{n+1} \gamma_{n+1}-\gamma_{n+1} r_{i}^{n} \\
& =1-\omega_{n+1} \gamma_{n}+\left(\omega_{n+1}-\gamma_{n}\right) r_{i}^{n} \\
& \leq 1-\omega_{n+1} \gamma_{n}+\left(\omega_{n+1}-\gamma_{n}\right) \gamma_{n} \\
& =-\left(\gamma_{n}^{2}-1\right)<0 . \\
& \Longrightarrow r_{3 i+1}^{n+1} \leq \gamma_{n} .
\end{aligned}
$$

Similarly, we get

$$
r_{3 i+2}^{n+1} \leq \gamma_{n}
$$

Combining Inequalities (22), (23) and (24), we have

$$
r_{i}^{n+1} \leq \gamma_{n}
$$


Similar manner, it can be verified that $\frac{1}{r_{3 i}^{n+1}} \leq \gamma_{n}, \frac{1}{r_{3 i+1}^{n+1}} \leq \gamma_{n}$ and $\frac{1}{r_{3 i+2}^{n+1}} \leq \gamma_{n}$. Thus, combining these inequalities, we get

$$
\frac{1}{r_{i}^{n+1}} \leq \gamma_{n}
$$

As, $R^{n+1}=\max _{i}\left\{r_{i}^{n+1}, \frac{1}{r_{i}^{n+1}}\right\}$,

$\therefore R^{n+1}<\gamma_{n}$.

Hence, by mathematical induction we get $d_{i}^{n}>0, R^{n}<\gamma_{n}$.

Now, we establish the convexity preserving condition in the limiting case, as $n \rightarrow \infty$. As the parameter $\omega_{n+1}$ given in Eq. (2) satisfies $\lim _{n \rightarrow \infty} \omega_{n+1}=2$. Consequently, $\lim _{n \rightarrow \infty} \gamma_{n}=\frac{7}{8}+\frac{1}{16} \sqrt{388}$ in Theorem 3 and note that the proof can be followed from Proposition 3.

Theorem 3. Suppose that the initial control points are strictly convex, such that,

$$
R^{0} \leq \frac{7}{8}+\frac{1}{16} \sqrt{388}
$$

then the limit curves generated by the subdivision scheme (1) are strictly convex.

\section{$6 \quad$ Numerical Applications}

In order to examine and certify the capability of positivity, monotonicity and convexity conditions that are suggested for the scheme (1), the following three numerical examples are executed.

Example 6.1. Comparison between binary 4-point non-stationary interpolating scheme of Beccari et al. [7] and ternary 4-point non-stationary interpolating scheme of Beccari et al. [1] after three iterations is well demonstrated in Figure (1). In the figure, the initial control polygons are shown by the doted lines, and the limit curves at $\omega_{0}=2$ for schemes [7] and [1] are shown by the green and red solid curves, respectively.

Example 6.2. For examining the applications of the scheme (1) after three iterations is well demonstrated in Figure (2). In the figure, the initial control polygons with five vertices are shown by the doted lines, and the limit curves at $\omega_{0}=2,2.5,3,10,15,50$ are shown by the green solid curves.

Example 6.3. For examining the applications of the scheme (1) after four iterations is well demonstrated in Figure (3). In the figure, the initial control polygons with five vertices are shown by the doted lines, and the limit curves at $\omega_{0}=2,2.5,3,10,15,50$ are shown by the blue solid curves.

Example 6.4. For examining the applications of the scheme (1) after three iterations is well demonstrated in Figure (4). In the figure, the initial control polygons with five vertices are shown by the doted lines, and the limit curves at $\omega_{0}=2,2.5,3,10,15,50$ are shown by the red solid curves. 


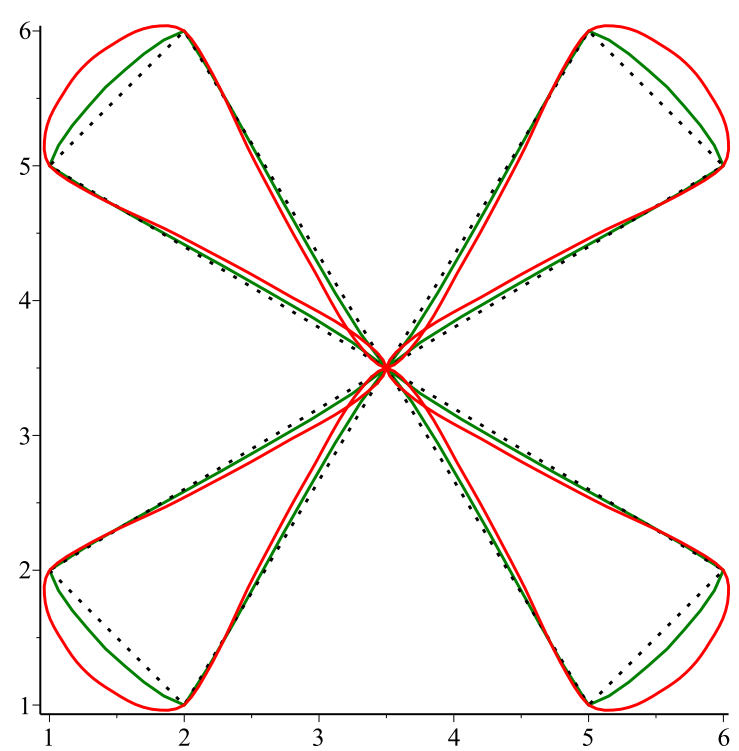

(a) $\omega_{0}=2$

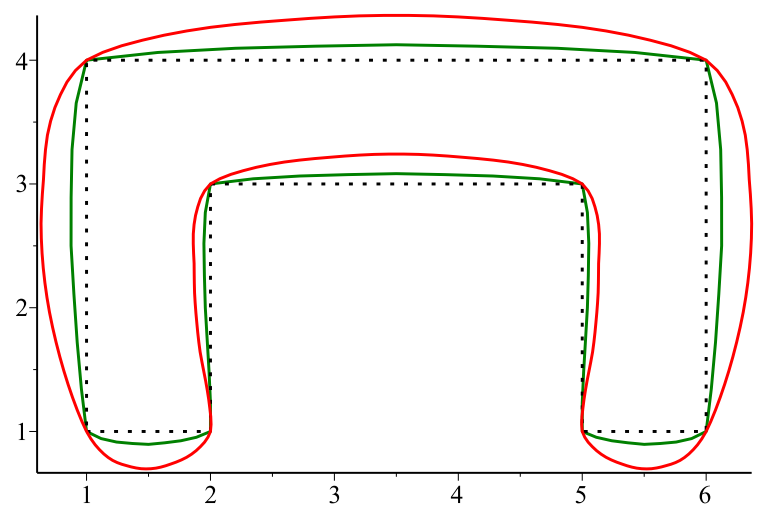

(b) $\omega_{0}=2$

Figure 1: In Figure (a) and (b) we show the behavior $C^{1}$-continuous limit curves generated after three iterations by applying the schemes in (1) and scheme [7] at $\omega_{0}=2$.

\section{Conclusion}

In this paper, we have been examined the shape preserving properties of 4-point binary nonstationary interpolating (1)subdivision scheme with the tension parameter $\omega$. The conditions on initial data are imposed, that if it is strictly convex and satisfy positivity, monotonicity and convexity, then subdivision scheme (1) preserve shape property. Some examples illustrate that our schemes have greater flexibility to geometric designers by choosing appropriate tension parameter. 


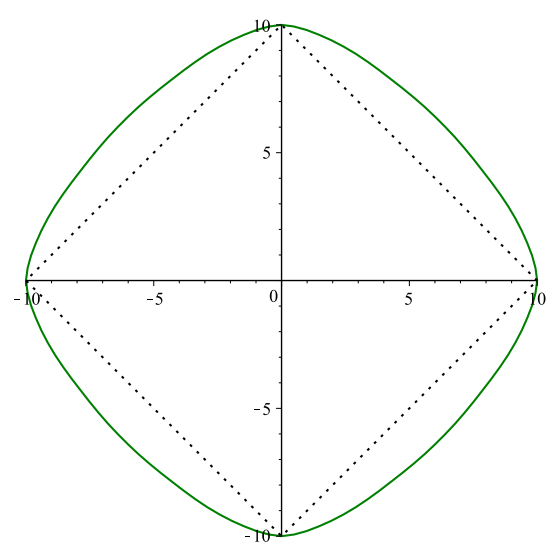

(a) $\omega_{0}=2$

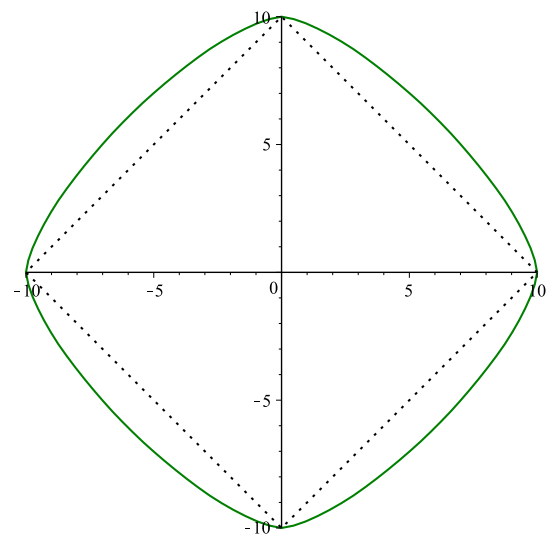

(c) $\omega_{0}=3$

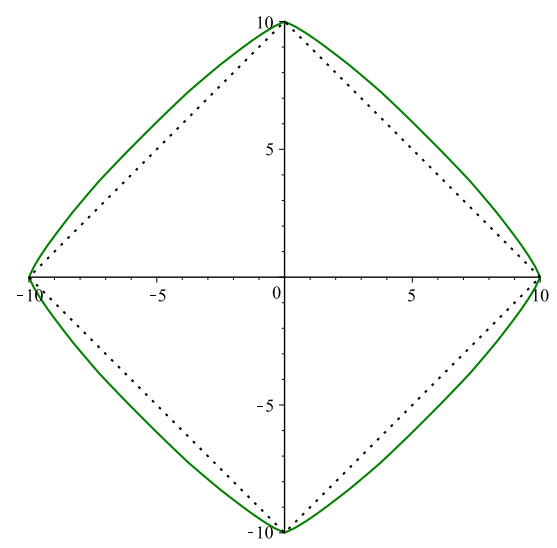

(e) $\omega_{0}=15$

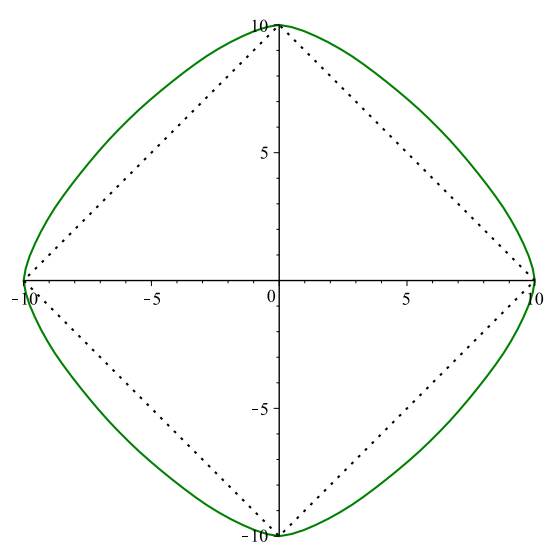

(b) $\omega_{0}=2.5$

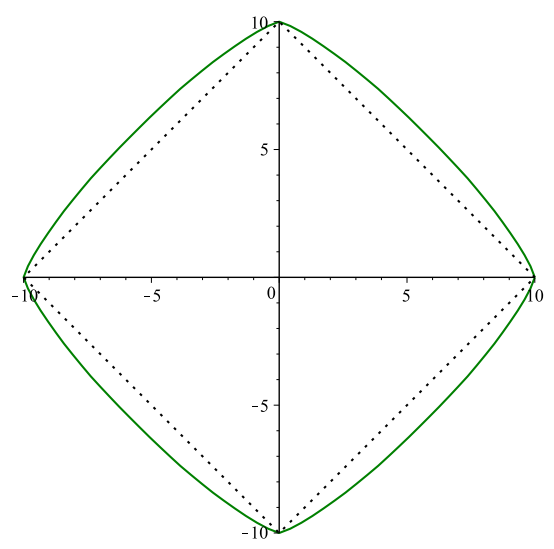

(d) $\omega_{0}=10$

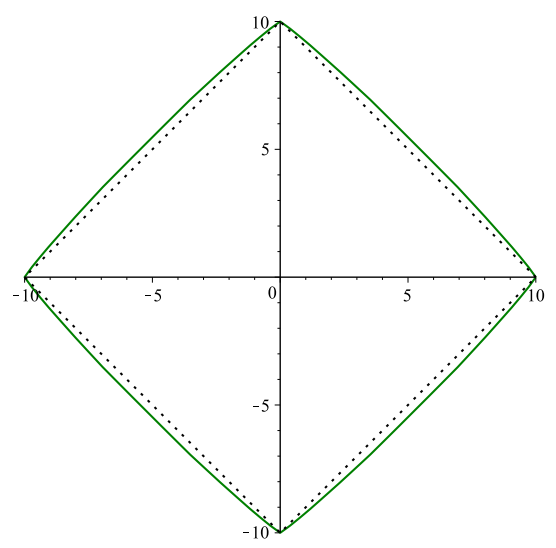

(f) $\omega_{0}=50$

Figure 2: From (a-f) we show the behavior $C^{1}$-continuous limit curves generated after three iterations by applying the scheme in (1) with $\omega_{0}=2,2.5,3,10,15,50$. 


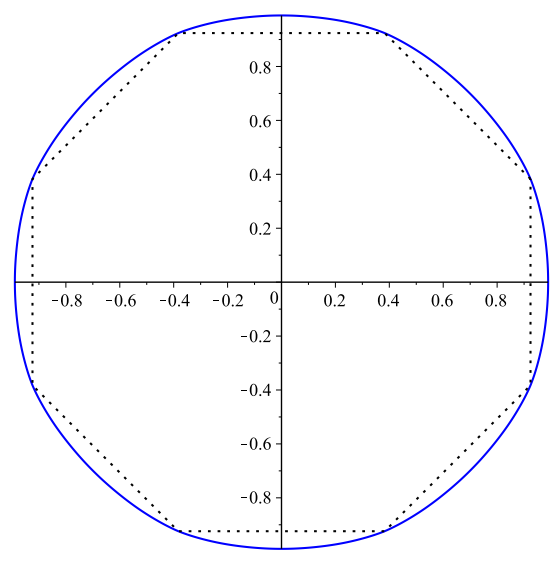

(a) $\omega_{0}=2$

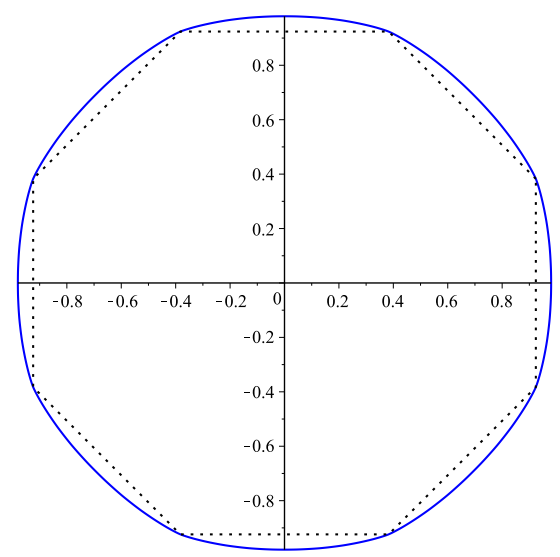

(c) $\omega_{0}=3$

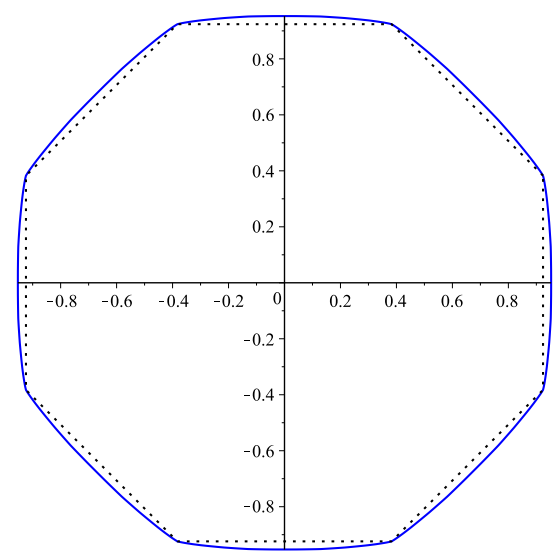

(e) $\omega_{0}=15$

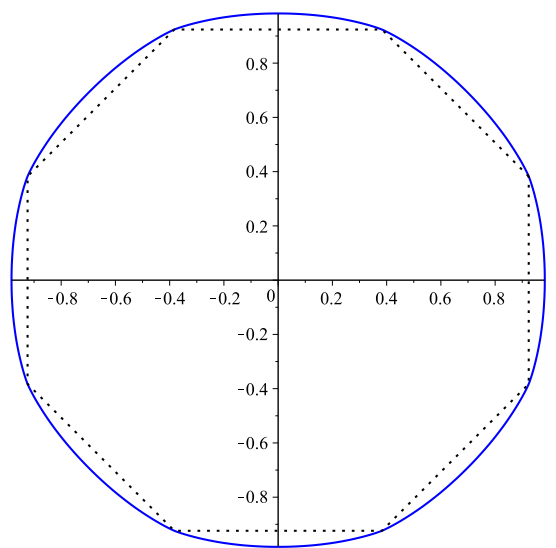

(b) $\omega_{0}=2.5$

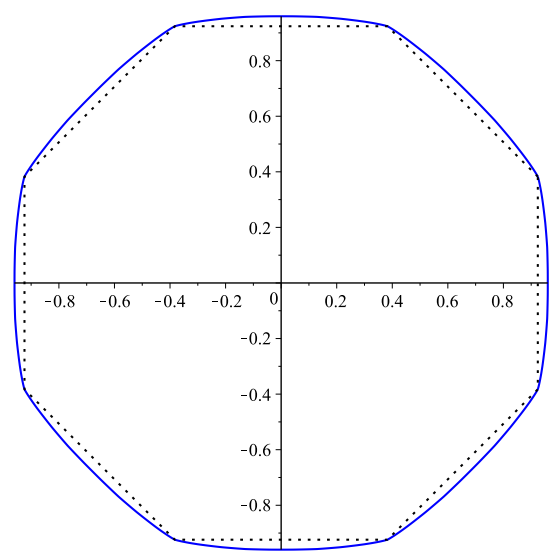

(d) $\omega_{0}=10$

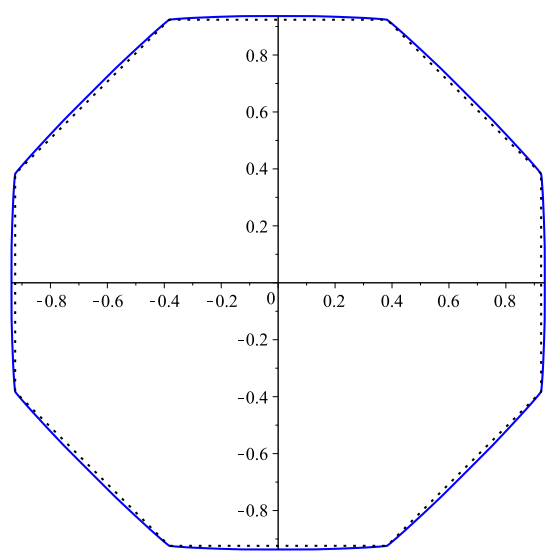

(f) $\omega_{0}=50$

Figure 3: From (a-f) we show the behavior $C^{1}$-continuous limit curves generated after four iterations by applying the scheme in (1) with $\omega_{0}=2,2.5,3,10,15,50$. 


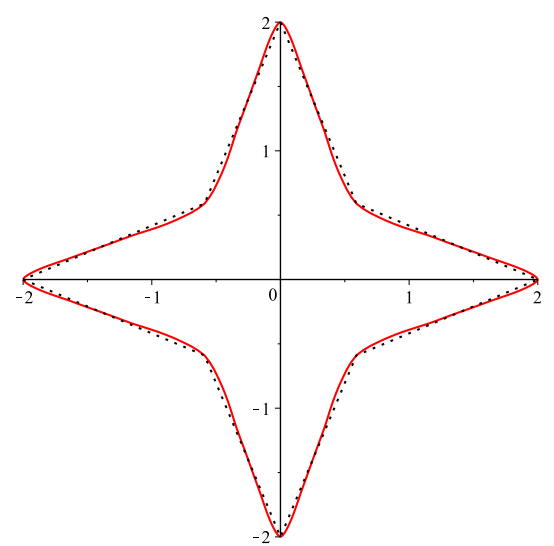

(a) $\omega_{0}=2$

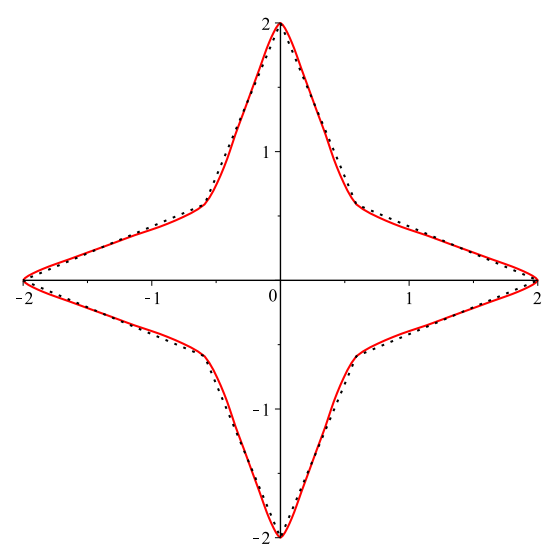

(c) $\omega_{0}=3$

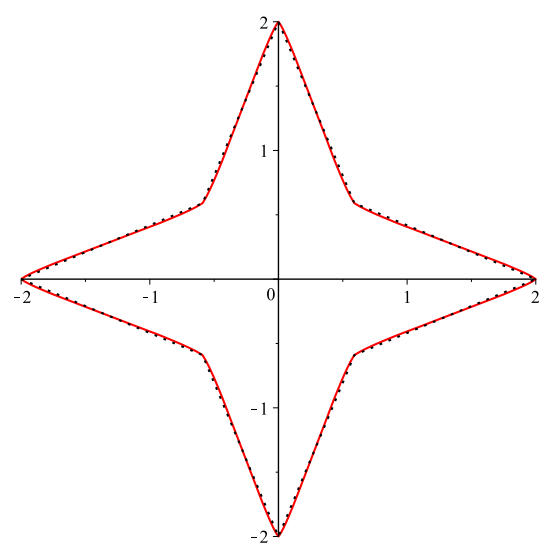

(e) $\omega_{0}=15$

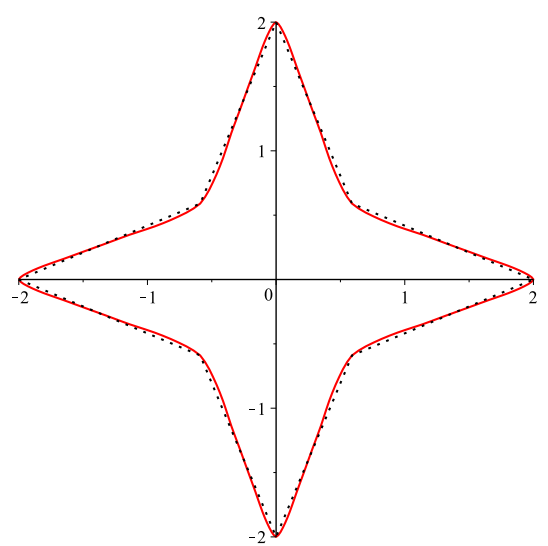

(b) $\omega_{0}=2.5$

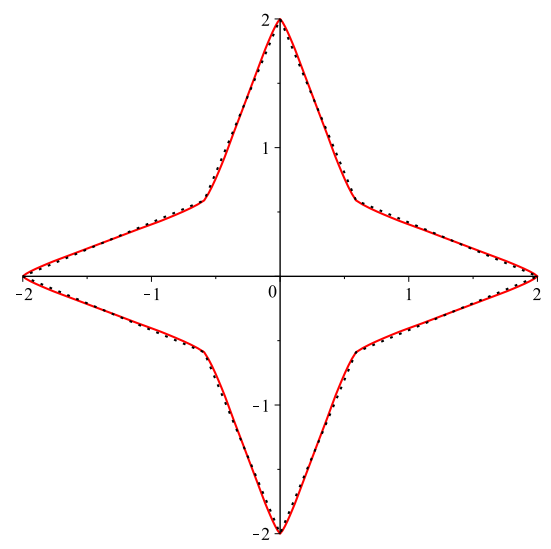

(d) $\omega_{0}=10$

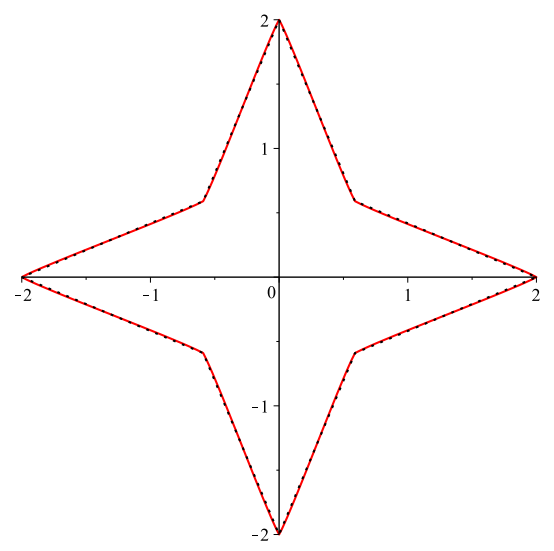

(f) $\omega_{0}=50$

Figure 4: From (a-f) we show the behavior $C^{1}$-continuous limit curves generated after four iterations by applying the scheme in (1) with $\omega_{0}=2,2.5,3,10,15,50$. 


\section{References}

[1] Beccari, C.; Casciola, G. \& Romani, L. Shape controlled interpolatory ternary subdivision Applied mathematics and computation, Elsevier, 2009, 215, 916927.DOI10.1016/j.amc.2009.06.014

[2] Romani, L. From approximating subdivision schemes for exponential splines to highperformance interpolating algorithms Journal of Computational and Applied Mathematics, Elsevier, 2009, 224, 383-396.

[3] Dyn, N. \& Levin, D. Stationary and non-stationary binary subdivision schemes Academic Press, 1992.

[4] Jena, M. K.; Shunmugaraj, P. \& Das, P. A subdivision algorithm for trigonometric spline curves Computer Aided Geometric Design, Elsevier, 2002, 19, 71-88.

[5] Jena, M. K.; Shunmugaraj, P. \& Das, P. A non-stationary subdivision scheme for generalizing trigonometric spline surfaces to arbitrary meshes Computer Aided Geometric Design, Elsevier, 2003, 20, 61-77.

[6] Beccari, C.; Casciola, G. \& Romani, L. An interpolating 4-point C2 ternary non-stationary subdivision scheme with tension control Computer Aided Geometric Design, Elsevier, 2007, 24, 210-219.

[7] Beccari, C.; Casciola, G. \& Romani, L. A non-stationary uniform tension controlled interpolating 4-point scheme reproducing conics Computer Aided Geometric Design, Elsevier, 2007, 24, 1-9.

[8] Daniel, S. \& Shunmugaraj, P. An interpolating 6-point C2 non-stationary subdivision scheme Journal of Computational and Applied Mathematics, Elsevier, 2009, 230, 164-172.

[9] Daniel, S. \& Shunmugaraj, P. Three point stationary and non-stationary subdivision schemes Geometric Modeling and Imaging, 2008. GMAI 2008. 3rd International Conference on, 2008, 3-8.

[10] Daniel, S. \& Shunmugaraj, P. Some non-stationary subdivision schemes Geometric Modeling and Imaging, 2007. GMAI'07, 2007, 33-38.

[11] Conti, C. \& Romani, L. A New Family of Interpolatory Non-Stationary Subdivision Schemes for Curve Design in Geometric Modeling AIP Conference Proceedings, 2010, 1281, 523-526.

[12] Conti, C. \& Romani, L. Algebraic conditions on non-stationary subdivision symbols for exponential polynomial reproduction Journal of Computational and Applied Mathematics, Elsevier, 2011, 236, 543-556.

[13] Siddiqi, S. S.; us Salam, W. \& Rehan, K. Binary 3-point and 4-point non-stationary subdivision schemes using hyperbolic function Applied Mathematics and Computation, Elsevier, $2015,258,120-129$. 
[14] Siddiqi, S. S.; us Salam, W. \& Rehan, K. A new non-stationary binary 6-point subdivision scheme Applied Mathematics and Computation, Elsevier, 2015, 268, 1227-1239.

[15] Bari, M. \& Mustafa, G. A Family of 2n-Point Ternary Non-Stationary Interpolating Subdivision Scheme Mehran University Research Journal of Engineering \& Technology, 2017, 36, 12.

[16] Tan, J.; Sun, J. \& Tong, G. A non-stationary binary three-point approximating subdivision scheme Applied Mathematics and Computation, Elsevier, 2016, 276, 37-43.

[17] Le MÂl'hautÃl', A. \& Utreras, F. I. Convexity-preserving interpolatory subdivision Computer Aided Geometric Design, Elsevier, 1994, 11, 17-37.

[18] Kuijt, F. \& van Damme, R. Convexity preserving interpolatory subdivision schemes Constructive approximation, Springer, 1998, 14, 609-630.

[19] Dyn, N.; Kuijt, F.; Levin, D. \& van Damme, R. Convexity preservation of the four-point interpolatory subdivision scheme Computer Aided Geometric Design, Elsevier, 1999, 16, 789-792.

[20] Dyn, N.; Levin, D. \& Gregory, J. A. A 4-point interpolatory subdivision scheme for curve design Computer aided geometric design, Elsevier, 1987, 4, 257-268.

[21] Cai, Z. Convexity preservation of the interpolating four-point C2 ternary stationary subdivision scheme Computer Aided Geometric Design, Elsevier, 2009, 26, 560-565.

[22] Yan, WANG \& Zhiming, LI A Family of Convexity-Preserving Subdivision Schemes Journal of Mathematical Research with Applications, 2017, 37, 489-495.

[23] Akram, G.; Bibi, K.; Rehan, K. \& Siddiqi, S. S. Shape preservation of 4-point interpolating non-stationary subdivision scheme Journal of Computational and Applied Mathematics, Elsevier, 2017, 319, 480-492.

[24] Dyn, N. \& Levin, D. Subdivision schemes in geometric modelling Acta Numerica, Cambridge University Press, 2002, 11, 73-144.

[25] Mustafa, G.; Ghaffar, A. \& Bari, M. (2n- 1)-point binary approximating scheme Digital Information Management (ICDIM), 2013 Eighth International Conference on, 2013, 363368.

[26] Daniel, S. \& Shunmugaraj, P. Some interpolating non-stationary subdivision schemes Computer Science and Society (ISCCS), 2011 International Symposium on, 2011, 400-403.

[27] Dyn, N.; Floater, M. S. \& Hormann, K. A C2 four-point subdivision scheme with fourth order accuracy and its extensions Analysis, , 1, f2.

[28] Daniel, S. \& Shunmugaraj, P. An approximating C2 non-stationary subdivision scheme Computer Aided Geometric Design, Elsevier, 2009, 26, 810-821. 
[29] Jena, M.; Shunmugaraj, P. \& Das, P. A non-stationary subdivision scheme for curve interpolation Anziam Journal, 2008, 44, 216-235. 